\title{
Improved Adsorption Capacity of EDTA-type Chitosan for Separation of Rare Earths
}

\author{
Motofumi MATSUdA $^{1 *}$ and Keisuke OHTO ${ }^{2}$ \\ ${ }^{1}$ SANTOKU Corporation., 4-14-34, Fukae-kitamachi, Higashinada-ku, Kobe-shi, Hyogo, 658-0013, Japan \\ ${ }^{2}$ Saga University, 1 Honjo-machi, Saga, 840-8502, Japan
}

\begin{abstract}
By optimizing the raw material ratio for the preparation of ethylenediaminetetraacetic acid-type chitosan (EDTA-type chitosan), the extraction efficiency of rare earth elements was improved. The synthesis with a lower concentration ratio of chitosan to acetic acid than that in the previously reported preparation method was confirmed by IR spectra that the peak assigned as the carboxyl group of EDTA was drastically decreased and the amide bond reaction between chitosan and EDTA was more facilitated. In the preparation method of this work, the amide formation was preferable to the competitive acetylation of chitosan. By the optimizing preparation conditions, the adsorption capacities for $\mathrm{Nd}, \mathrm{Sm}, \mathrm{Tb}$, and Dy were improved about twice. On the contrary, the separation factor of rare earths was not changed, and it is suggested that other compounds having adsorption ability were not produced. EDTA-type chitosan was applied to chromatographic separation of $\mathrm{La}, \mathrm{Nd}, \mathrm{Sm}, \mathrm{Tb}$, and $\mathrm{Dy}$. In the column method, it was confirmed that lighter rare earth sequentially eluted from the column, therefore it has been proved that adsorption of lighter rare earth was substituted by heavier rare earth.
\end{abstract}

\section{Introduction}

Nowadays, rare earth elements (REEs) are essential for many industrial products such as motors, semiconductors, abrasives, catalysts and so on. In particular, the use of rare earth magnets for motors has rapidly increased, and the demand will further increase for the spread of electric vehicles in near future. Mining and production of REEs are highly geographically distributed (U.S. Geological Survey, 2019). From view point of stable supply of REEs, recycling from REEs containing scrap is one of effective methods. In order to widely spread the recycling of REEs, the high separation performance for REEs and the small environmental load are required. At present, solvent extraction is widely used for the separation of REEs (Abdeltawab et al., 2002), however large-scale operation is limited due to the use of a large amount of flammable solvents such as kerosene.

To reduce the environmental load without using organic solvents, it has been reported that an extractant is combined or impregnated with an ion exchange resin (Shibata et al., 1994a; Shibata et al., 1994b; Nishihama et al., 2013). However, commercially available ion exchange resins are often very expensive and they have no functional groups available for binding with the extractant, and elution of the extractant from the impregnated matrix is a problem (Shibata et al., 1994b; Nishihama et al., 2013).
Chitosan is a natural resource derived from shrimp and crab shellfish. It is industrially prepared from by removing other components by treatment with acid and alkalis hydroxide solution. Chitosan also has a large number of active primary amino groups, so that various chemical modifications are possible. Inoue et al. (1995) synthesized ethylenediaminetetraacetic acid-type chitosan (EDTA-type chitosan) and found that EDTA were still functional after immobilization on chitosan. EDTA is a typical compound to form chelate and recover metal cations, and has been used to separate REEs (Byrne et al., 1995). Although column separation of REEs using EDTA-type chitosan is similarly used for separation as ion exchange method using EDTA as eluent (James et al., 1961), in ion exchange resin method, rare earth ions in solution forms stable chelates. Therefore, the conventional process for separation and recovery from the metal-loaded solution requires EDTA eluent. On the other hand, in using EDTA-type chitosan method, the eluent can be present as an acidic solution. Thus simplification of the process after separation can also be expected.

EDTA-type chitosan has reported which prepared in the almost same method and material ratio up to recent years (Repo et al., 2010). However, in this method, an excess amount of anhydrous EDTA was used as a raw material with respect to chitosan. There is room for investigation on the optimal reaction conditions for preparing EDTA-type chitosan.

\footnotetext{
*Corresponding author: mmatsuda@santoku-corp.co.jp
} 
In this study, the raw material ratio for the preparation of EDTA-type chitosan was changed and column separation of REEs using EDTA-type chitosan was operated.

\section{Experimental}

\subsection{Preparation of EDTA-type chitosan}

EDTA-type chitosan was prepared according to the scheme of Figure 1 by the following method (Inoue et al., 1995) that subsequently identified herein as "EC". $10.5 \mathrm{~g}$ of acetic acid (Guaranteed Reagent, FUJIFILM Wako Pure Chemical Corporation) was dissolved in $90 \mathrm{~mL}$ of distilled water and $5.0 \mathrm{~g}$ of chitosan (Y.H.chitosan KII, YAEGAKI Bio-industry, Inc.) was dissolved and diluted with methanol by 5 times volume. Thirty grams of ethylenediaminetetraacetic dianhydride (EDTA) (Tokyo Chemical Industry Co., Ltd.) suspended in $100 \mathrm{~mL}$ of methanol was added and the mixture was stirred at room temperature for about $24 \mathrm{~h}$. The precipitate was filtered and stirred in ethanol for $12 \mathrm{~h}$. After filtration again, the mixture was stirred with $\mathrm{NaOH}$ solution of $\mathrm{pH}=11$ for 12 $\mathrm{h}$ to remove unreacted EDTA. Thereafter, decantation was repeated with $0.1 \mathrm{M} \mathrm{HCl}$ and ion-exchanged water until the washing water became neutral. After filtration, it was dried in vacuo to obtain $6.7 \mathrm{~g}$ of a gel-like white solid.

The raw material ratio of EDTA-type chitosan was modified that subsequently identified herein as "ECG" (Table 1). Two and half grams of acetic acid was dissolved in $50 \mathrm{~mL}$ of distilled water and $5.0 \mathrm{~g}$ of chitosan was dissolved and diluted $150 \mathrm{~mL}$ of methanol. Three grams of EDTA suspended in $15 \mathrm{~mL}$ of methanol was added and stirred, then the mixture was turned into a gel in a few minutes. The gel was cut into about $5 \mathrm{~mm}$ pieces, and stirred in $3 \mathrm{wt} \% \mathrm{NaOH}$ solution for $1 \mathrm{~h}$ and left to stand for about $12 \mathrm{~h}$. Washing and drying processes were carried out as same in EC.
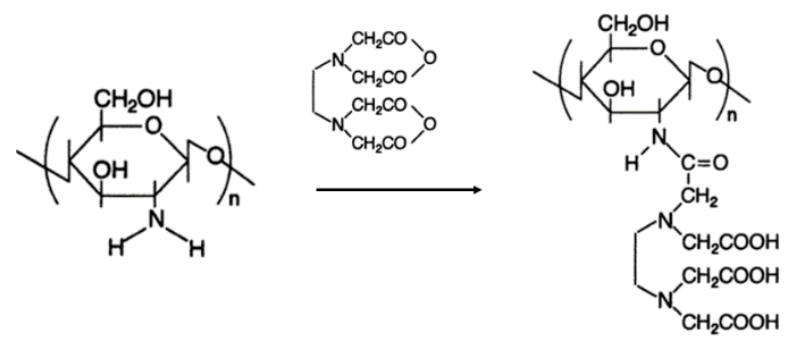

Figure 1. Synthetic route of EDTA-type chitosan

Table 1. Comparison of raw material ratio of EDTA-type chitosan

\begin{tabular}{cccccc}
\hline & $\begin{array}{c}\text { Methanol } \\
(\mathrm{mL})\end{array}$ & $\begin{array}{c}\text { Chitosan } \\
(\mathrm{g})\end{array}$ & $\begin{array}{c}\text { Water } \\
(\mathrm{mL})\end{array}$ & $\begin{array}{c}\text { Acetic acid } \\
(\mathrm{g})\end{array}$ & $\begin{array}{c}\text { EDTA } \\
\text { dianhydride }(\mathrm{g})\end{array}$ \\
\hline EC & 500 & 5.0 & 90 & 10.5 & 30 \\
ECG & 165 & 5.0 & 50 & 2.5 & 3.0 \\
\hline
\end{tabular}

\subsection{FTIR spectra}

FTIR spectra of the obtained samples in $\mathrm{KBr}$ disc were measured in a Shimadzu IR Affinity $1 . \mathrm{KBr}$ discs were prepared in the usual way from dried a few $\mathrm{mg}$ of samples and about $100 \mathrm{mg}$ of $\mathrm{KBr}$.

\subsection{Batchwise adsorption}

Aqueous solutions were prepared by dissolving each metal chloride salt to a concentration of $0.1 \mathrm{mmol} / \mathrm{L}$ in 0.1 $\mathrm{mol} / \mathrm{L} \quad \mathrm{HCl}$ and $0.1 \mathrm{~mol} / \mathrm{L}$ HEPES $(\mathrm{N}-(2-$ hydroxyethyl)piperazin- $N$ '-(2-ethanesulfonic acid)) buffer solution. Both solutions contained $\mathrm{Nd}, \mathrm{Sm}, \mathrm{Tb}$, and Dy ions. These aqueous solutions were mixed to adjust $\mathrm{pH}=1.3$. Seven and half milliliters of aqueous phase and $10 \mathrm{mg}$ of EDTA-type chitosan were mixed and shaken for $16 \mathrm{~h}$ at $30^{\circ} \mathrm{C}$. After filtration, the metal ion concentrations were measured by inductively coupled plasma atomic emission spectrophotometer (ICP-AES, Shimadzu, ICPS$8100)$. The rate of extraction $(\% E)$, amount of metal ion adsorbed $\left(q, \mathrm{kmol} \mathrm{kg}^{-1}\right)$, and distribution ratio $\left(D, \mathrm{dm}^{3} \mathrm{~kg}\right.$ ${ }^{-1}$ ) are defined by Eqs. (1), (2) and (3):

$$
\begin{aligned}
& \% E=100 \times\left(C_{i}-C_{e}\right) / C_{i} \\
& q=\left(C_{i}-C_{e}\right) \mathrm{V} / 1000 \mathrm{~W} \\
& D_{L n}=1000 \mathrm{q} / C_{e}
\end{aligned}
$$

where $C_{i}$ and $\left.C_{e}(\mathrm{~mol} \mathrm{dm})^{-3}\right)$ represent the initial and equilibrium concentrations of the metal ion in the aqueous phase, and $V(\mathrm{~mL})$ and $W(\mathrm{~g})$ are the volume of aqueous solution and weight of EDTA-type chitosan, respectively. The amount of adsorption of each metal was determined from the difference between the metal concentration before and after adsorption and the dry weight of the adsorbent according to equation (2), and the distribution ratio was further determined according to equation (3).

\subsection{Chromatographic separation of rare earths}

ECG was applied to chromatographic separation of $\mathrm{La}, \mathrm{Nd}, \mathrm{Sm}, \mathrm{Tb}$, and Dy. Aqueous solutions were prepared by dissolving each metal chloride salt to a concentration of $0.2 \mathrm{mmol} / \mathrm{L}$ in $0.1 \mathrm{~mol} / \mathrm{L} \mathrm{HCl}$ and $0.1 \mathrm{~mol} / \mathrm{L}$ HEPES buffer solution. Both solutions contained $\mathrm{La}, \mathrm{Nd}, \mathrm{Sm}, \mathrm{Tb}$, and Dy ions. These aqueous solutions were mixed to adjust $\mathrm{pH}=1.3$. Three milliliters of pre-swollen ECG (dry weight $0.23 \mathrm{~g}$ ) soaked in $\mathrm{pH}=2.0$ of $\mathrm{HCl}$ was packed into a chromatographic-tube with $12 \mathrm{~mm}$ diameter and 200 $\mathrm{mm}$ height. The aqueous feed solution was fed downwards from tightly capped top of the tube at flow rate of $0.5 \mathrm{~mL} / \mathrm{min}$ (space velocity, S.V.=10 $\mathrm{h}^{-1}$ ), using peristaltic tubing pump (Fisherbrand CON3385). Passed liquid was collected for every $1 \mathrm{~mL}$. The metal ion concentrations were measured by ICP-AES. 


\section{Results and Discussion}

\subsection{FTIR spectroscopy}

Figure 2 shows the IR spectra of (a) ECG, (b) EC, and (c) raw chitosan. In both of obtained samples ((a) and (b)), the band at approximately $1680 \mathrm{~cm}^{-1}$ that is assigned to carbonyl of the amide bond involved in EDTA-type chitosan are observed.

In the sample (a) ECG, the band at $1736 \mathrm{~cm}^{-1}$ assigned to carboxyl group of EDTA and ester groups is drastically decreased compared to (b) EC. It was indicated that the amide bond reaction between chitosan and EDTA was more progressed by the optimizing preparation conditions.

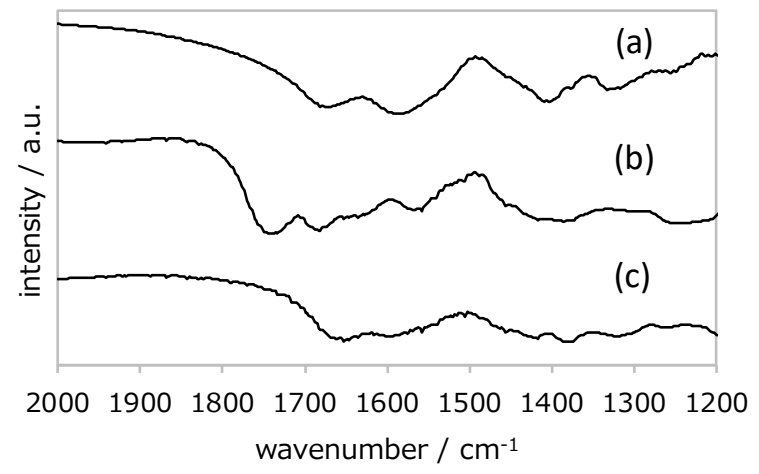

Figure 2. IR spectra of (a) ECG, (b) EC, and (c) raw chitosan

\subsection{Batchwise adsorption}

Figure 3 shows extraction efficiency of $\mathrm{Nd}, \mathrm{Sm}, \mathrm{Tb}$, and Dy using EC and ECG. The extraction efficiency was improved about twice for ECG compared to EC. This is the effect of optimizing preparation of EDTA-type chitosan by EC and ECG.

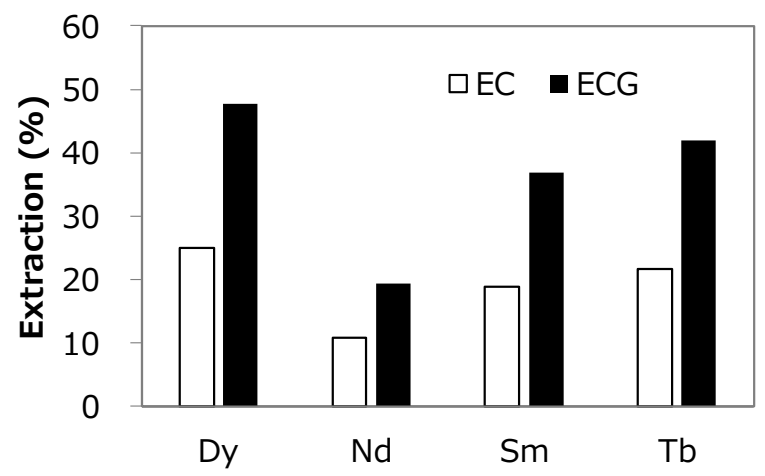

Figure 3. Effect of optimizing preparation of EDTA-type chitosan on extraction of rare earths by EC and ECG

On the contrary, Figure 4 shows that the separation factor $\left(D_{L n 1} / D_{L n 2}\right)$ of REEs by EC and ECG. Separation factor was calculated by the combination of distribution ratio $\left(D_{L n}\right)$ of Dy-Tb, Dy-Nd and Nd-Sm. There was no difference in the separation factors for pairs of REEs between EC and ECG. This suggests that EDTA component significantly contributes to the REEs adsorption. It is considered that other compounds having adsorption ability were not produced.

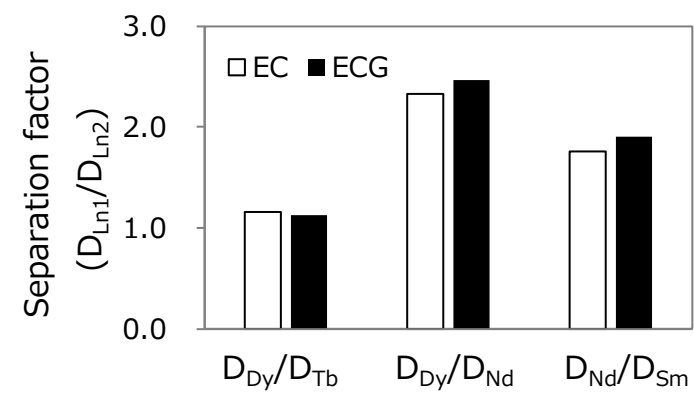

Figure 4. Effect of optimizing preparation of EDTA-type chitosan on extraction of rare earths by EC and ECG

It is reported that chitosan is $N$-acetylated in acetic acid methanol - water system (Hirano et al., 1976; Moore et al., 1980). In the preparation method of this work, the binding reaction between chitosan and anhydrous EDTA competes with the acetylation of chitosan, but it is thought that the binding reaction with anhydrous EDTA could be facilitated by optimazing the raw material ratio. In the previous report (Moore et al., 1980), many parameters that contribute to the acetylation of chitosan have been reported. Although it is difficult to identify the main factor that made the reaction with anhydrous EDTA advantageous, It is presumed that lowering the concentration of acetic acid used as the raw material is one of the factors (Table 1).

\subsection{Chromatographic separation of rare earths}

Figure 5 shows breakthrough curves for $\mathrm{Nd}, \mathrm{Sm}, \mathrm{Tb}$, and Dy from a metal solution with EDTA-type chitosan packed column. Light REEs begin to leak out of the column first because they have a lower affinity for EDTA than heavy REEs. The concentration of the effluent solution of $\mathrm{La}$ is partially higher than the inlet concentration, since La once adsorbed on EDTA-type chitosan was extruded by the exchange reaction with heavier elements in the feed solution. It is possible to collect high-purity $\mathrm{La}$ by fractionating the effluent solution up to the breakthrough point of Nd. Heavy REEs can also be separated by adjustment of the acid concentration at the elution process. 


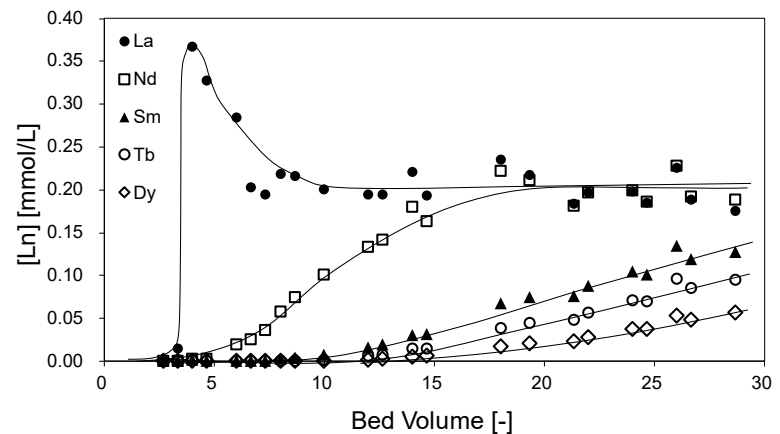

Figure 5. Breakthrough curves for $\mathrm{Nd}, \mathrm{Sm}, \mathrm{Tb}$, and $\mathrm{Dy}$ from a metal solution with EDTA-type chitosan packed column. Feed concentrations of Nd, Sm, Tb, and Dy are $0.2 \mathrm{mmol} / \mathrm{L}$ each. $\mathrm{pH}$ feed $=1.3, \mathrm{~S} . \mathrm{V} .=10 \mathrm{~h}^{-1}$

\section{Conclusions}

By the synthesis with a lower concentration ratio of chitosan to acetic acid than that in the previously reported preparation of EDTA-type chitosan, the amide formation between chitosan and EDTA was more facilitated.

The extraction efficiency of REEs are improved about twice by the optimizing preparation conditions. And other compounds having adsorption ability were not produced, while separation factor was not drastically changed.

In the column method, it has been proved that adsorption of lighter rare earth was substituted by heavier rare earth. By setting various conditions such as the filling amount and the flow rate effectively, mutual separation of neighboring element can be expected, which is promising as a separation method with low environmental load.

\section{Acknowledgements}

This research was financially supported by NEDO project "Research and Development of Recycling Technologies for Establishing a High Efficiency Resource Circulation System".

\section{References}

Abdeltawab, A. A., S. Nii, F. Kawaizumi, and K. Takahashi; "Separation of La and Ce with PC-88A by Counter-Current Mixer-Settler Extraction Column," Sep. Pur. Technol., 26, 265-272 (2002)

Byrne, R. H. and B. Li; "Comparative Complexation Behavior of the Rare Earths," Geochim. Cosmochim. Acta, 59, 4575-4589 (1995)

Hirano, S. and R. Yamaguchi; "N-acetylchitosan gel: A Polyhydrate of Chitin," Biopolymers, 15, 1685-1691 (1976)
Inoue, K., K. Ohto, K. Yoshizuka, R. Shinbaru, and K. Kina; "Adsorption Behaviors of Some Metal Ions on Chitosan Modified with EDTA- Type Ligand," BUNSEKI KAGAKU, 44, 283-287 (1995)

James, D. B., J. E. Powell, and F. H. Spedding; "Cationexchange Elution Sequences-I Divalent and Rare-Earth Cations with EDTA, Hedta and Citrate," J.-Inorg. Nucl. Chem., 19, 133-141 (1961)

Moore, G. K. and G. A. F. Roberts; "Chitosan Gels: 1. Study of Reaction Variables," Int. J. Biolog. Macromol., 2, 73-77 (1980)

Nishihama, S., K. Kohata, and K.Yoshihara; "Separation of Lanthanum and Cerium Using a Coated SolventImpregnated Resin," Sep. Pur. Technol, 118, 511-518 (2013)

Repo, E., J. K. Warchol, T. A. Kurniawan, M. E. T. Sillanpää; "Adsorption of $\mathrm{Co}(\mathrm{II})$ and $\mathrm{Ni}(\mathrm{II})$ by EDTAand/or DTPA-modified Chitosan: Kinetic and Equilibrium Modelling," Chem. Eng. J., 161, 73-82 (2010)

Shibata, J., T. Nagasaka, and M. Mashimo; "Separation of $\mathrm{Nd}$ and Sm by Column Method," Shigen-to-Sozai, 110, 191-194 (1994a)

Shibata, J., M. Mashimo, and T. Nagasaka; "Separation of Tb, Dy and Ho by Column Method," Shigen-to-Sozai, 110, 613-617 (1994b)

U.S. Geological Survey, Mineral Commodity Summaries 2019, p. 132 (2019)

https://www.usgs.gov/centers/nmic/mineral-commoditysummaries 